Journal of Animal and Veterinary Advances 11 (19): 3557-3560, 2012

ISSN: $1680-5593$

(C) Medwell Journals, 2012

\title{
Development of Algorithm to Detect Presence of Animal in Digital Image
}

\author{
Boon Tatt Koik and Haidi Ibrahim \\ School of Electrical and Electronic Engineering, Engineering Campus, \\ University Sains Malaysia, 14300 Nibong Tebal, Penang, Malaysia
}

\begin{abstract}
Animal detection can be used in wide range of applications especially intrusion detection of animal for security reason to ensure the safety of human life and assets. In this study, an image processing method is proposed using multiple image frames comparison approach based on background subtraction. A simple background subtraction followed by predefined threshold filtration to filter the noises of natural image is performed to obtain a rough output, then multiple images pixels data in Boolean expression are compared to reduce false positive detection of animal is performed. Connected component and region labelling operation being implemented to group and classify detected area region followed by area threshold's operation to further eliminate unwanted anomalies in image frames. The detected animal in images will be marked with rectangle frame.
\end{abstract}

$\underline{\text { Key words: Animal detection, background subtraction, morphology operation, threshold, filtration, Malaysia }}$

\section{INTRODUCTION}

The researches on animal detection have been a major topic for various applications. Animal detection methods are useful on the research on locomotive behavioural of targeted animal and also to prevent dangerous animal intrusion in residential area. In this study, target extraction by using Threshold Segmentation Method, connected component labelling, morphology and detection of animal in images in the form of framing is performed.

Target extraction from background can be performed by using Threshold Segmentation Method. In Chenyang et al. (2007), the object is found by using Background Subtraction Method after obtaining the background image. In Nascimento and Marques (2006), Threshold Segmentation Method based on the pixel values is performed. However, in this technique, researchers should carefully choose the threshold value as they also should consider the negative value obtained at certain pixel point by direct subtraction.

The idea of threshold segmentation is simple which pixel that greater than threshold are set to white (i.e., intensity 255) and those less than the threshold value will be set to black (i.e., intensity 0). As stated in Nascimento and Marques (2006), it is difficult to select the threshold accurately as the background image periodically changes. Therefore, different appropriate threshold should be chosen for different background scene.

There are a lot of problems need to be considered in developing an animal detection algorithm. First is the lighting problem in which a sudden change of lighting effect mostly in indoor application can affect the effectiveness in detecting the presence of animal intrusion. Besides, luminance problem with changes of natural environment from day to night at outdoor surveillance system can also affect the detection. Furthermore, moving background such as leaves by wind might be regarded as foreground image and some inactive animal which remain static for a long time can be mistakenly interpreted as background image by the algorithms (Storring et al., 2001). In this study, multiple pictures frames have been taken as a sample in a continuous time interval which can be regarded as a stream of video that has been chopped into many picture frames. After threshold segmentation of images, morphology opening and closing are performed to improve the images. This process is important for next step which is connected component labelling as too many noises and distorted animal images will affect the performance of connected component algorithms.

Researchers use a simple and efficient connected component algorithm (Di Stefano and Bulgarelli, 1999) rather than a more complex algorithm in Samet and Tamminen (1986) and Dillencourt et al. (1992) as algorithm

Corresponding Author: Boon Tatt Koik, School of Electrical and Electronic Engineering, Engineering Campus, 
in Di Stefano and Bulgarelli (1999) is sufficient for an accurate animal detection. Area threshold been applied after connected component labelling to eliminate anomalies in images. In this study, many filtration processes has been performed as unlike indoor images, outdoor natural images have lots of noise and animal might covered partly by background to reduce false positive detection of multiple animals in images. Lastly, detected animal in the image will be indicated by a frame in original images.

\section{MATERIALS AND METHODS}

First stage (Subtraction and threshold filtration): For the implementation of animal detection in multiple frames of images, background subtraction method has been implemented to compare multiple frames of images. Pixel of the same coordination will be subtracted with each in image frame and store as absolute value. The subtracted pixel value will be set to 255 (white) whenever the threshold exceeds a predefined value (Fig. 1).

Multiple image frames comparison: The produced black and white image will be further filtered by comparing with each other image again to reduce false positive detection of animal. For a sample data consists of 4 images, comparison will be done just like Fig. 2:

Image 1-Image 2; Image 1-Image 3; Image 1-Image 4

Image 2-Image 3; Image 2-Image 4; Image 3-image 4

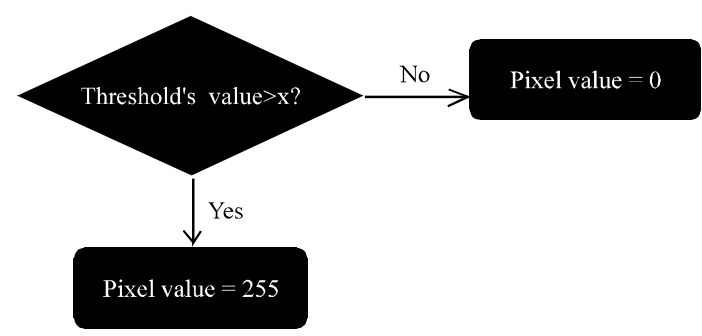

Fig. 1: Subtraction and threshold filtration

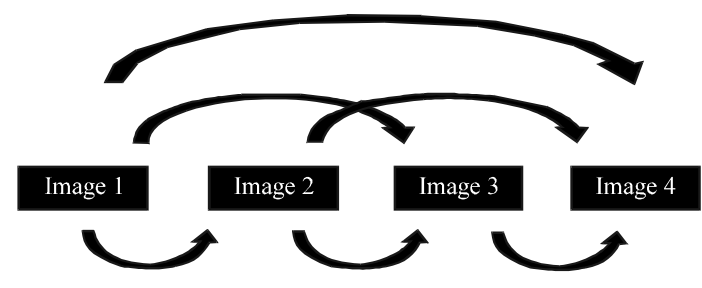

Fig. 2: Multiple image frames comparison
Four the 4 images of data sample, 6 comparisons will be done and the value will be stored as Boolean constants true or false. The 6 Boolean constants will be compared as a group, 3 Boolean constant at one time as:

$$
\left.\begin{array}{l}
\text { Image 1-Image 4-Image 5; Image 1-Image 2-Image } 3 \\
\text { Image 2-Image 3-Image-5; Image 3-Image 5-Image } 6
\end{array}\right\}
$$

Second stage (Morphological operation): The processed images will undergo morphological operation to further improve detected animal image and reduce any unnecessary noise inimage frame. Morphological operation is essential especially in this, i.e. as we focus on natural scene (outdoor) where there are lots of noise, e.g., movement of tree by wind and animal might partly covered by plant. For morphological operation, a loop of erosion process on pixel image value 255 will be implemented. Morphological opening implemented witherosion process, followed by dilation while for morphological closing, dilation process followed by erosion. The purpose of closing operation is to solidify any empty pixel region within detected animal image while opening operation is to further filter out anomalies in image frames processed. The morphological operation will be carried out as in Eq. 1 (Roomi et al., 2010; Erring et al., 2001):

$$
\text { Dilation }_{\mathrm{xy}}=(\mathrm{X} \oplus \mathrm{Y}) ; \text { Erosion }_{\mathrm{ij}}=(\mathrm{I} \ominus \mathrm{J})
$$

Third stage (Connected component method and region labeling: The purpose of connected component method is to group the nearby pixel together to form an exclusive area region. In this method, the surrounding 8 pixels of an individual pixel will be processed to see if there is similar pixel around it and grouping will be done on that detected region.

In first scan, 8 connectivity of each pixel surrounding been processed to produce exclusive region to differentiate one area to the other in images. Second scan detect the nearby region of same intensity for merging to produce less scattered region in images. For first scan:

$$
\left.\begin{array}{l}
\operatorname{pixel}_{i, j} \text { will becompared with } \\
\operatorname{pixel}_{i+1, j}, \operatorname{pixel}_{i-1, j}, \operatorname{pixel}_{i+1, j-1}, \operatorname{pixel}_{i-1, j-1} \\
\operatorname{pixel}_{i+1, j+1}, \operatorname{pixel}_{i-1, j+1}, \operatorname{pixel}_{i+1, j}, \operatorname{pixel}_{i, j+1}, \operatorname{pixel}_{i, j+1}
\end{array}\right\}
$$

Fourth stage (Area threshold filtration and detection of animal): Area threshold filtration is to further filter out anomalies other than detected animal. Researchers make an application of fuzzy logic if area $>$ thr, then pixel value in area $=0$ if area $<$ thr then pixel value $=0$. Lastly, a red 
frame will be drawn to the detected animal (white region) starting from top left of pixel to lowest right pixel of the detected region (Fig. 3).

Further processes and application can be further carried out after the detected animal in image frame such as turning on video to higher definition for clearer locomotive research and triggering alarm for further action as intrusion prevention purpose.

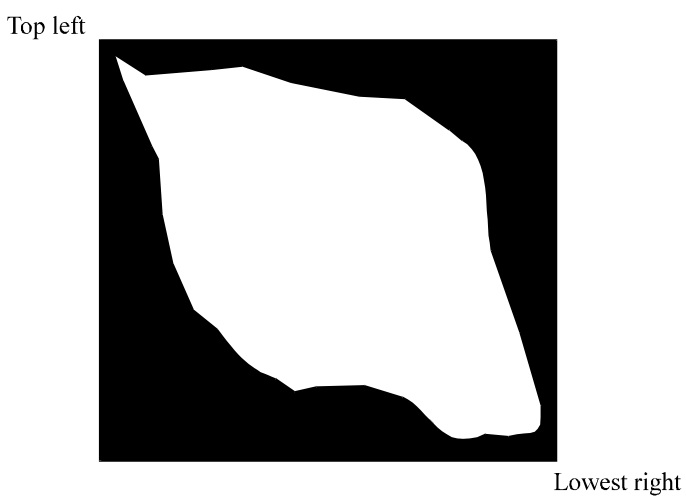

Fig. 3: Detection of animal

\section{RESULTS AND DISCUSSION}

Animal detection (Fig. 4a-d) are sample data obtained after background subtraction and threshold's processes followed by multiple image frames comparison. Researchers can notice that there is a distinct animal object in the image (distinct white area). Animal detection (Fig. 4e-h) are processed data obtained after morphology, connected component region labelling and area threshold filtration. Notice that all the noises in natural scene have been filtered and the detected region of animal become more distinct in the images.

Animal detection (Fig. 4i-1) are the result images for animal detection, a rectangle frame is drawn to the detected animal in images. Animal detection(Fig. 4m-p) are the result images for another sample data of animal detection at close distance.

Animal detection (Fig. 4a-d) notice that there is lots of noise in the images. These happen as natural images e.g., plants always moving caused by wind in open area. Animal detection (Fig. 4e-h) notice that the animal are from quite a far distant from the camera and relatively
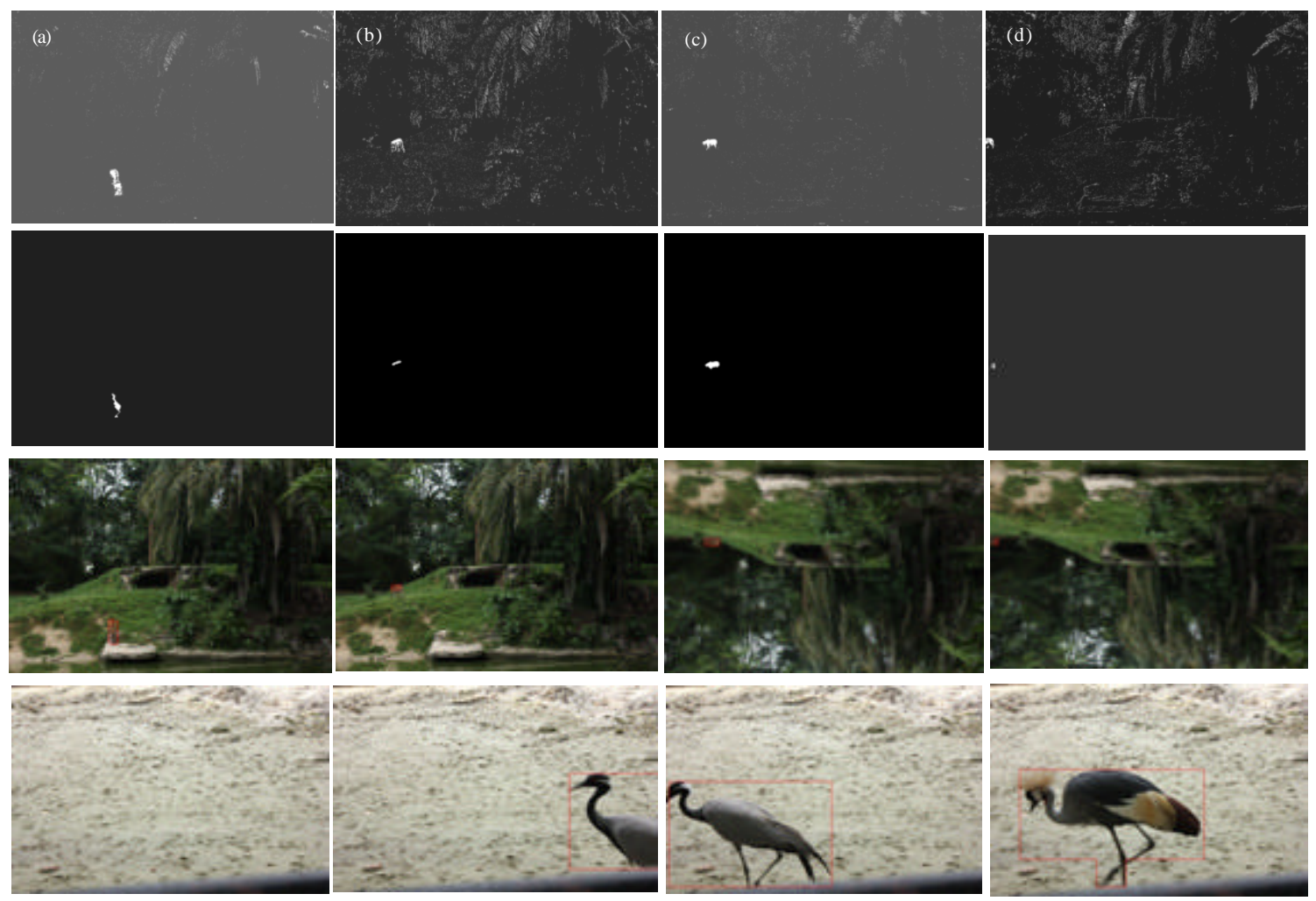

Fig. 4: Animal detection 
small compared to close distance caption therefore, researchers can say that this animal detection algorithm still effective for long distance detection. Animal detection (Fig. 4i-1) show the detected animal from far distance while figures: animal detection (Fig. 4m-p) shows the detection from close distance. From data, researchers can say that the animal detection algorithm is suitable for detection for far and close distance of animal in images.

The combination of frames in figure: animal detection (Fig. 4p) to produce a single frame has prevented a single animal being detected as two. In short, this study has successfully detected the presence of animal in digital images.

\section{CONCLUSION}

The proposed method can work in both low and high quality images its potential to be applied in real life animal intrusion detection scenario is very high.

\section{ACKNOWLEDGEMENTS}

This research was supported in part by the Universiti Sains Malaysia's Short Term Research Grant with account number 304/PELECT/60311013 and Universiti Sains Malaysia's Research University Grant with title Development of Wide Secured Wireless Sensor Network (WSN) with Unmanned Aerial Vehicle (UAV) Deployment for the Monitoring of Ecology and Global Warming Studies Towards Environment Sustainability.

\section{REFERENCES}

Chenyang, W., Z. Mingquan and G. Guohua, 2007. Moving object detection based on background model. Comput. Technol. Dev., 17: 21-23.

Di Stefano, L. and A. Bulgarelli, 1999. A simple and efficient connected components labeling algorithm. Proceedings of the International Conference on Image Analysis and Processing, September 27-29, 1999, IEEE Computer Society, Washington DC., USA., pp: 322-327.

Dillencourt, M.B., H. Samet and M. Tamminen, 1992. A general approach to connected component labeling for arbitrary image representations. J. ACM, 39: 253-280.

Nascimento, J.C. and J.S. Marques, 2006. Performance evaluation of object detection algorithms for video surveillance. IEEE Trans. Multimedia, 8: 761-774.

Roomi, S.M.M., P. Rajesh, R.J. Priya and M. Senthilarasi, 2010. A line model based approach for monkey intrusion detection. Proceedings of the International Conference on Signal Processing and Communications, Bangalore, July 18-21, 2010, IEEE Computer Society, Washington DC. USA., pp: 1-5.

Samet, H. and M. Tamminen, 1986. An improved approach to connected component labeling of images. Proceedings of the IEEE Conference on Computer Vision and Pattern Recognition, June 1986, IEEE Computer Society, USA., pp: 312-318.

Storring, M., H.J. Andersen and E. Granum, 2001. Physicsbased modelling of human skin colour under mixed illuminants. Robot. Autonom. Syst., 35: 131-142. 